\title{
Visibility-Based Hypothesis Testing Using Higher-Order Optical Interference
}

\author{
Michał Jachura, ${ }^{1,}{ }^{*}$ Marcin Jarzyna, ${ }^{2}$ Michał Lipka, ${ }^{1}$ Wojciech Wasilewski, ${ }^{1}$ and Konrad Banaszek ${ }^{1,2}$ \\ ${ }^{1}$ Faculty of Physics, University of Warsaw, Pasteura 5, 02-093 Warszawa, Poland \\ ${ }^{2}$ Centre of New Technologies, University of Warsaw, Banacha 2c, 02-097 Warszawa, Poland
}

(Received 31 October 2017; published 16 March 2018)

\begin{abstract}
Many quantum information protocols rely on optical interference to compare data sets with efficiency or security unattainable by classical means. Standard implementations exploit first-order coherence between signals whose preparation requires a shared phase reference. Here, we analyze and experimentally demonstrate the binary discrimination of visibility hypotheses based on higher-order interference for optical signals with a random relative phase. This provides a robust protocol implementation primitive when a phase lock is unavailable or impractical. With the primitive cost quantified by the total detected optical energy, optimal operation is typically reached in the few-photon regime.
\end{abstract}

DOI: 10.1103/PhysRevLett.120.110502

Optical systems, in addition to being the workhorse of modern telecommunication, provide a natural platform to implement quantum-enhanced protocols for information transfer and processing between distant parties. Quantum strategies can provide authentication or reduce the communication complexity of certain tasks, in which large distributed data sets need to be processed to infer a relatively small amount of information [1,2]. Examples include quantum digital signatures [3] and quantum fingerprinting [4]. These protocols share a primitive that consists in imprinting the input data onto the modal structure of transmitted fields, e.g., in the form of phase patterns, and interfering the received signals, as shown in Fig. 1. Different hypotheses, e.g., the instances of identical and unequal inputs, are mapped onto distinct ranges of the interference visibility, which can therefore serve as the basis for hypothesis testing. Strikingly, optical signals sufficient to realize the quantum scheme may not have the capacity to carry information necessary to implement the classical protocols with the matching confidence level. This enhancement, stemming from the interplay between the wave and particle properties of light exploited in quantum protocols, can advantageously change the scaling of resources required to perform the task as well as ensure security.

As recently pointed out $[5,6]$ and demonstrated experimentally [7-10], the protocol primitive described above can be realized efficiently with coherent light beams and first-order interference. This implementation uses laser light sources and is robust against attenuation introduced by optical channels transmitting the signals, but it requires phase stability between the sending parties. In certain scenarios a shared phase reference may be unavailable or very difficult to furnish. An alternative may be to resort to Hong-Ou-Mandel interference between single photons, which has been exploited in proof-of-principle demonstrations of quantum communication complexity protocols [11,12]. However, a practical implementation may require single photon sources with long coherence times and would be inefficient for high channel attenuation. The latter impairment affects also a realization based on weak classical states with a random global phase [13].

In this Letter we present a strategy to carry out optical hypothesis testing based on the visibility of higherorder interference between classical fields with a random relative phase. This approach concurrently benefits from conventional optical signal generation techniques, removes the need for a shared phase reference, and ensures robustness against channel attenuation. The performance is

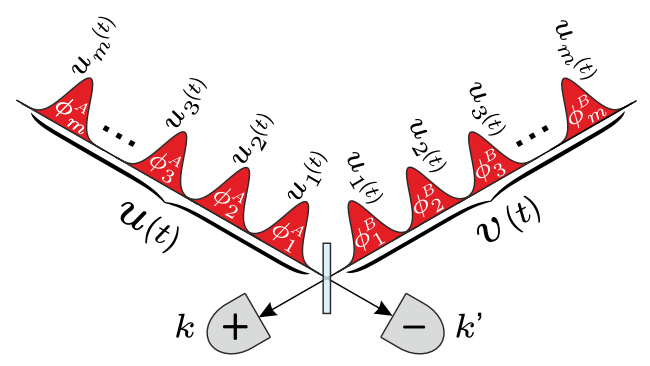

FIG. 1. An interferometric primitive for visibility-based hypothesis testing with phase-keyed signals. Input data in possession of two parties $A$ and $B$ are mapped onto phase patterns $\phi_{1}^{A}, \phi_{2}^{A}, \ldots, \phi_{m}^{A}$ and $\phi_{1}^{B}, \phi_{2}^{B}, \ldots, \phi_{m}^{B}$ used to modulate sequences of $m$ pulses described by a family of normalized temporal waveforms $u_{1}(t), u_{2}(t), \ldots, u_{m}(t)$. Generated optical signals can be viewed as prepared in collective modes described, respectively, by $u(t)=$ $\sum_{j=1}^{m} u_{j}(t) \exp \left(i \phi_{j}^{A}\right) / \sqrt{m}$ and $v(t)=\sum_{j=1}^{m} u_{j}(t) \exp \left(i \phi_{j}^{B}\right) / \sqrt{m}$. The signals are brought to interference at a 50:50 beam splitter whose output ports are monitored by photodetectors. The outcome of a single repetition of an interferometric measurement is a pair of integers $k, k^{\prime}$ specifying the number of counts registered by each of the detectors over the duration of the signals. 
characterized using average error probability, whose asymptotic behavior is investigated with the help of a refined Chernoff bound [14,15]. Interestingly, we show that when the protocol cost is quantified in terms of the total transmitted optical energy, the optimal strategy is to realize multiple repetitions of the interference visibility measurement in the few-photon regime with a determination of the complete photocount statistics.

Let us first consider interference between two mutually coherent optical signals. Each signal has the form of a pulse sequence depicted in Fig. 1 and carries optical energy $\bar{n} / 2$ expressed in photon number units. The receiver combines the signals on a balanced beam splitter. The time-integrated light intensity at the two output ports of the beam splitter labeled with indices "+" and "-" can be written as $I^{ \pm}(\mathcal{V})=\eta \bar{n}(1 \pm \operatorname{Re} \mathcal{V}) / 2$, where $\eta$ is the channel transmission for each of the signals [16]. Here, $\mathcal{V}$ is the interference visibility that carries information about the relation between the input data sets. In the ideal case it is equal to the overlap $\mathcal{V}=\int d t u(t) v^{*}(t)$ between the normalized waveforms $u(t)$ and $v(t)$ describing the two received signals. For identical inputs $\mathcal{V}=1$, which corresponds to completely destructive interference at the output port. Hence, registering a photocount at that port unambiguously indicates that the inputs were unequal. This observation underpins the quantum fingerprinting protocol, which aims at deciding whether data sets in possession of two parties are identical or different while revealing the smallest possible amount of information to the external referee. The protocol employs classical error correction to guarantee that for any pair of unequal inputs the visibility remains below a certain threshold value. Given that experimental imperfections, such as detector dark counts and misalignment of optical beams, lower the effective visibility [16], the hypotheses of identical or unequal inputs correspond to two distinct ranges of the visibility parameter separated by a gap. In order to perform a practical test between these two hypotheses one needs to devise a decision rule based on the measured photocount statistics.

Let the detectors at the output ports of the beam splitter be able to resolve up to $K$ photocounts over the signal duration. The probability $p_{k}^{ \pm}$of registering $k$ photocounts on one detector reads $p_{k}^{ \pm}(\mathcal{V})=\exp \left[-I^{ \pm}(\mathcal{V})\right]\left[I^{ \pm}(\mathcal{V})\right]^{k} / k$ ! for $k=0,1, \ldots, K-1$ and $p_{K}^{ \pm}(\mathcal{V})=1-\sum_{k=0}^{K-1} p_{k}^{ \pm}(\mathcal{V})$. Nonunit efficiency of the detectors can be included in the channel transmission $\eta$. Suppose now that the signal pairs are received with a promise that the visibility takes only one of two equiprobable values $\mathcal{V}_{1}$ or $\mathcal{V}_{2}$. For the fingerprinting protocol one value corresponds to identical inputs, while the second one can be taken as the highest visibility occurring in the case of unequal inputs. The task is to discriminate between the two visibility hypotheses on the basis of the photocount sample collected in $N$ repetitions of the interferometric measurement. The probability $\varepsilon$ of erroneously identifying the actual visibility is upper bounded by the so-called Chernoff bound $\varepsilon \leq \exp (-N C) / 2$ [14], where $C$ stands for the Chernoff information given explicitly by

$C=-\log \left[\min _{0 \leq \alpha \leq 1}\left(\sum_{k, k^{\prime}=0}^{K}\left[P_{k k^{\prime}}\left(\mathcal{V}_{1}\right)\right]^{\alpha}\left[P_{k k^{\prime}}\left(\mathcal{V}_{2}\right)\right]^{1-\alpha}\right)\right]$.

In the above expression, the summation is carried out over all possible measurement outcomes, which in our setup have the form of two integers $k$ and $k^{\prime}$ specifying the number of counts registered by individual detectors, and $P_{k k^{\prime}}(\mathcal{V})$ denotes the probability of obtaining a specific combination $k k^{\prime}$ for the visibility $\mathcal{V}$.

For the coherent signal scenario considered so far, the probability of registering, respectively, $k$ and $k^{\prime}$ counts has the product form $P_{k k^{\prime}}^{\text {coh }}(\mathcal{V})=p_{k}^{+}(\mathcal{V}) p_{k^{\prime}}^{-}(\mathcal{V})$. Assuming full photon number resolution with $K \rightarrow \infty$, the Chernoff information can simplified to

$$
\begin{aligned}
C^{\mathrm{coh}}= & \eta \bar{n}\left(1-\frac{1}{2} \min _{0 \leq \alpha \leq 1}\left[\left(1+\operatorname{Re} \mathcal{V}_{1}\right)^{\alpha}\left(1+\operatorname{Re} \mathcal{V}_{2}\right)^{1-\alpha}\right.\right. \\
& \left.\left.+\left(1-\operatorname{Re} \mathcal{V}_{1}\right)^{\alpha}\left(1-\operatorname{Re} \mathcal{V}_{2}\right)^{1-\alpha}\right]\right)
\end{aligned}
$$

It is seen that the Chernoff information is proportional to the received optical energy $\eta \bar{n}$. The proportionality factor given by the ratio $C^{\mathrm{coh}} /(\eta \bar{n})$ can be interpreted as the amount of information gained from the detection of one photon. In Fig. 2(a) we depict this factor as a function of the real parts of visibilities $\operatorname{Re} \mathcal{V}_{1}$ and $\operatorname{Re} \mathcal{V}_{2}$. Generally, it pays off to maintain a large distance between the visibilities with the maximum information attained for $\mathcal{V}_{1}=-\mathcal{V}_{2}= \pm 1$.

The above picture becomes much more nuanced if the sending parties have no access to a shared phase reference, which implies that the signals arrive with a random relative phase. However, in each individual realization the signals are described by coherent waveforms whose overlap is given by $\mathcal{V}$ up to an overall phase factor. In such a scenario, the joint photocount distribution reads

$$
P_{k k^{\prime}}^{\mathrm{rnd}}(\mathcal{V})=\int_{0}^{2 \pi} \frac{d \varphi}{2 \pi} p_{k}^{+}\left(e^{i \varphi} \mathcal{V}\right) p_{k^{\prime}}^{-}\left(e^{i \varphi} \mathcal{V}\right)
$$

The explicit analytical expression for $P_{k k^{\prime}}^{\text {rnd }}(\mathcal{V})$ is derived in the Supplemental Material [16]. Obviously, after averaging over the global phase only the absolute value $|\mathcal{V}|$ of the visibility parameter is relevant. The above probability distribution can be used to calculate the respective Chernoff information $C^{\text {rnd }}$ according to Eq. (1). As before, the ratio $C^{\text {rnd }} /(\eta \bar{n})$ has the interpretation of the amount of information gained per one received photon.

In Fig. 3 we depict $C^{\text {rnd }} /(\eta \bar{n})$ as a function of the received optical energy $\eta \bar{n}$ for an exemplary pair of visibilities $\mathcal{V}_{1}=0.98$ and $\mathcal{V}_{2}=0.56$. The linear scaling 

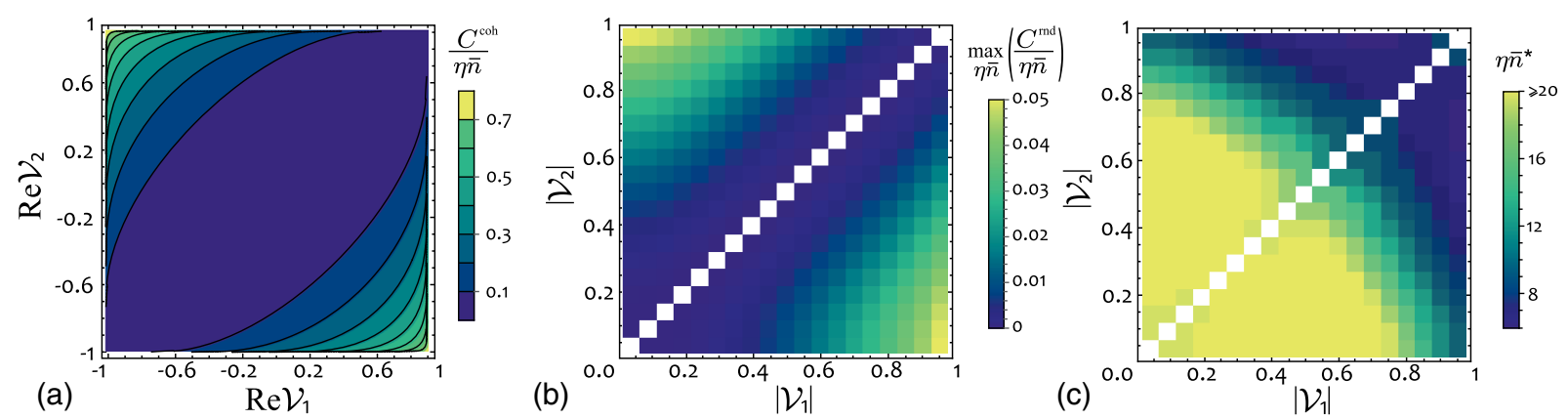

FIG. 2. Discrimination between a pair of hypotheses encoded in interference visibilities $\mathcal{V}_{1}, \mathcal{V}_{2}$ for the coherent and the random-phase scenario. (a) Information gained from a single photodetection event for a fixed phase between interfering signals. (b) Maximum information per one detected photon $C^{\text {rnd }} /(\eta \bar{n})$ for signals with a random global phase. (c) Optimal $\eta \bar{n}^{*}$ maximizing the ratio $C^{\text {rnd }} /(\eta \bar{n})$. White squares on the diagonal in (b) and (c) represent the case $\left|\mathcal{V}_{1}\right|=\left|\mathcal{V}_{2}\right|$ when the two hypotheses are indistinguishable.

of the ratio $C^{\text {rnd }} /(\eta \bar{n})$ with $\eta \bar{n}$ for $\eta \bar{n} \ll 1$ is explained by the fact that for very weak signals a detection of at least two photons in a single realization of the measurement is necessary to obtain any meaningful information [13]. Consequently, in this regime the leading term of the Chernoff information $C^{\text {rnd }}$ is proportional to $(\eta \bar{n})^{2}$, which gives unfavorable quadratic scaling with the channel transmission. Beyond the two-photon regime corresponding to low optical energies, the ratio $C^{\text {rnd }} /(\eta \bar{n})$ exhibits a well pronounced maximum in $\eta \bar{n}$. This observation can be used to draw the following operational conclusion. Suppose that the total optical energy available at transmitters is $\bar{n}_{\text {tot }}$. If $\bar{n}$ photons are used in a single realization of the interferometric measurement, one can afford $N=\bar{n}_{\text {tot }} / \bar{n}$ repetitions. Let us rewrite the Chernoff bound on the error probability as $\exp \left(-N C^{\text {nnd }}\right) / 2=\exp \left[-\eta \bar{n}_{\text {tot }} C^{\text {nnd }} /(\eta \bar{n})\right] / 2$. Assuming a fixed $\bar{n}_{\text {tot }}$, which can be taken as the overall cost of implementing the communication primitive, it is beneficial to optimize $C^{\text {rnd }} /(\eta \bar{n})$ for a single realization.

Remarkably, the optimum of $C^{\text {rnd }} /(\eta \bar{n})$ occurs for $\eta \bar{n}$ in the few-photon range and information needed for

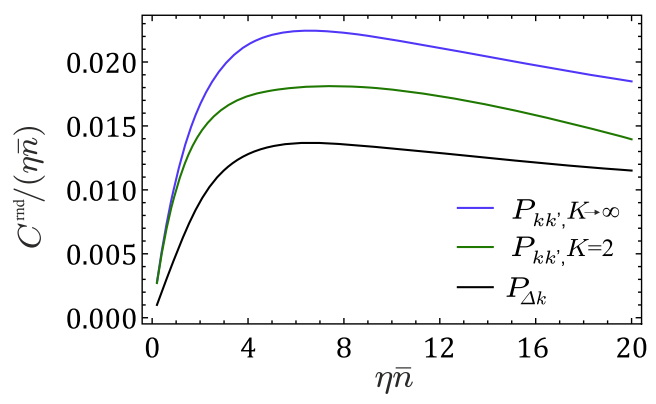

FIG. 3. Chernoff information per one detected photon $C^{\text {rnd }} /(\eta \bar{n})$ as a function of the average photon number $\eta \bar{n}$ in a single realization of the visibility measurement with a random global phase, depicted for the pair of visibilities $\mathcal{V}_{1}=0.98$ and $\mathcal{V}_{2}=0.56$. The scenario based on full photocount statistics $K \rightarrow \infty$ (blue line) is compared with limited photon number resolution $K=2$ (green line), and inference based only on the photocount difference $\Delta k=k^{\prime}-k$ (black line). hypothesis testing is distributed in a nontrivial manner across the entire joint photocount statistics. To illustrate this point, in Fig. 3 we depict also the noticeably lower ratio $C^{\text {tnd }} /(\eta \bar{n})$ calculated for detection that could resolve only up to $K=2$ photocounts over the signal duration. Further, using only the marginal distribution for the photocount number difference $P_{\Delta k}(\mathcal{V})=\sum_{k} P_{k, k+\Delta k}(\mathcal{V})$ reduces significantly the Chernoff information, as also shown in Fig. 3. The above observations are universal as long as one of the two visibilities is sufficiently high, which is the case of quantum protocols motivating this study. In Fig. 2(b) we plot the maximum $C^{\text {rnd }} /(\eta \bar{n})$ as a function of the absolute values of the visibilities $\left|\mathcal{V}_{1}\right|$ and $\left|\mathcal{V}_{2}\right|$ to be discriminated between, along with the optimal average photon number that should be used in a single realization shown in Fig. 2(c). Generally, the amount of Chernoff information per unit optical energy is lower than in the coherent scenario depicted in Fig. 2(a), which is easily explained by the lack of the phase reference. Nevertheless, the available information also scales linearly with the optical energy, which implies that the scaling advantage over classical protocols should be analogous to the coherent case.

We performed a proof-of-principle experimental demonstration of binary hypothesis testing for a pair of visibilities $\mathcal{V}_{1}=0.98$ and $\mathcal{V}_{2}=0.56$ using a collinear interferometric setup presented in Fig. 4(a). We employed a continuous-wave $800 \mathrm{~nm}$ laser diode attenuated by a series of neutral-density filters down to $\approx 10^{-14} \mathrm{~W}$ of power followed by a polarizer ensuring a well-defined linear polarization. The beam is subsequently sent through a combination of a quarter-wave and a half-wave plate whose respective rotation angles $\theta$ and $\phi$ define the normalized intensities after the Wollaston polarizer as $I^{ \pm}=\left\{1 \pm \operatorname{Re}\left[e^{4 i \phi-2 i \theta} \cos (2 \theta)\right]\right\} / 2$. Hence, our experimental setup can be viewed as a fully equivalent simulation of a standard interferometer with the complex visibility tunable in the entire phase and absolute value range by an appropriate rotation of the wave plates. To realize the random phase scenario we collected data for 50 half-wave 


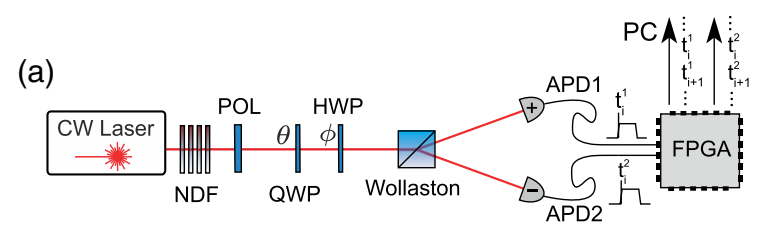

(b)

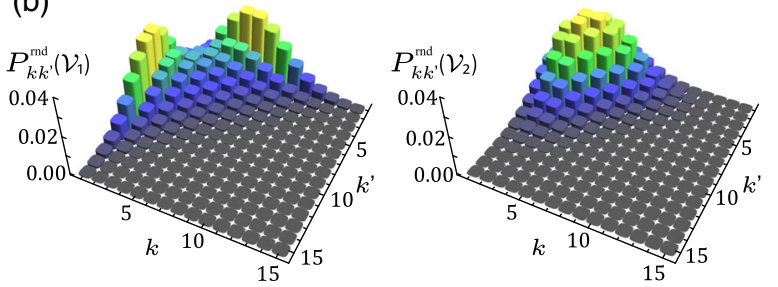

(c)

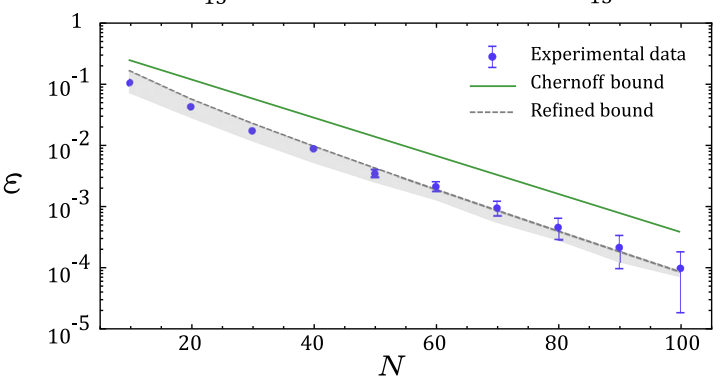

FIG. 4. (a) The simplified scheme of the experimental setup. NDF, neutral density filters. POL, polarizer. HWP, half-wave plate. QWP, quarter-wave plate. APD, avalanche photodiode. FPGA, time tagger based on field-programmable gate array. (b) Experimental joint photocount distributions $P_{k k^{\prime}}^{\text {rnd }}$ obtained from approximately $1.5 \times 10^{6}$ outcomes $k k^{\prime}$ for visibilities $\mathcal{V}_{1}=0.98$ (left), $\mathcal{V}_{2}=0.56$ (right). (c) The error probability $\varepsilon$ in hypothesis testing as a function of the data set size $N$, determined for each $N$ from $1.5 \times 10^{4}$ repetitions of the Neyman-Pearson test on independent sets of experimental data. The results are compared with the standard Chernoff bound (green, solid line) and its refined version (gray, dashed line). The error bars account for 1 standard deviation. The gray shaded region corresponds to the error probability of testing hypotheses $\mathcal{V}_{1}=0.98$ vs $\mathcal{V}_{2}$ from the range $0 \leq \mathcal{V}_{2} \leq 0.56$ using the Neyman-Pearson test designed for $\mathcal{V}_{2}=0.56$.

plate angles $\phi$ uniformly probing a full period of the visibility phase. Both output beams were monitored by free-running avalanche photodiodes connected to a time tagger based on a field-programmable gate array architecture, which registered photocounts with $3.3 \mathrm{~ns}$ temporal resolution.

The time tagged counts for each of the two detectors were grouped over $80-\mu$ s-long time intervals. With the used input power, this interval corresponds to the mean photocount number $\eta \bar{n}=6.3$, which gives the partitioning of the total optical energy that nearly maximizes information per one detected photon. The numbers of photodetection events accumulated over an individual interval yield the single realization outcome $k k^{\prime}$. The $50 \mathrm{~ns}$ dead time of the detectors used in the setup did not noticeably distort the measured photon statistics. We collected approximately $1.5 \times 10^{6}$ pairs $k k^{\prime}$ for each of the two visibilities. This allowed us to determine the joint probability distributions $P_{k k^{\prime}}^{\text {rnd }}\left(\mathcal{V}_{1,2}\right)$ depicted in Fig. 4(b), which within the resolution of the graphs match perfectly the theoretical values given by Eq. (3). A detailed analysis is presented in the Supplemental Material [16].

In order to experimentally determine the error probability of binary hypothesis testing one needs to repeat the test procedure multiple times feeding it with independent sets of experimental data obtained for a fixed visibility. We realized this by selecting from the experimental results an ensemble of $M=1.5 \times 10^{4}$ data sets $\left[\left(k k^{\prime}\right)_{1}, \ldots,\left(k k^{\prime}\right)_{N}\right]_{1}$, $\left[\left(k k^{\prime}\right)_{1}, \ldots,\left(k k^{\prime}\right)_{N}\right]_{2}, \ldots,\left[\left(k k^{\prime}\right)_{1}, \ldots,\left(k k^{\prime}\right)_{N}\right]_{M}$ consisting of $N$ photocount pairs. We applied the Neyman-Pearson test [14] to each data set selecting as the test result the visibility yielding a higher likelihood of photocount group observation, i.e., $\mathcal{V}_{1}$ if $\prod_{i=1}^{N} P_{\left(k k^{\prime}\right)_{i}}^{\mathrm{rnd}}\left(\mathcal{V}_{1}\right)>\prod_{i=1}^{N} P_{\left(k k^{\prime}\right)}^{\mathrm{rnd}}\left(\mathcal{V}_{2}\right)$ and $\mathcal{V}_{2}$ otherwise. The probability of error was evaluated as the ratio of erroneous hypothesis determinations to the number of groups $M$ used for testing. That way we estimated the conditional error $\varepsilon\left(\mathcal{V}_{1} \mid \mathcal{V}_{2}\right)$ of inferring visibility $\mathcal{V}_{1}$ when $\mathcal{V}_{2}$ was the true one and the reverse error $\varepsilon\left(\mathcal{V}_{2} \mid \mathcal{V}_{1}\right)$.

In Fig. 4(c) we compare the average error probability determined from experimental data $\varepsilon=\left[\varepsilon\left(\mathcal{V}_{1} \mid \mathcal{V}_{2}\right)+\right.$ $\left.\varepsilon\left(\mathcal{V}_{2} \mid \mathcal{V}_{1}\right)\right] / 2$ with both the standard Chernoff bound for the random phase scenario and the refined Chernoff bound [15] derived explicitly in the Supplemental Material [16]. In accordance with theoretical predictions, the experimental error remains below the upper bound provided by the Chernoff bound [14], reaching its refined version for an asymptotically large number $N$ of outcomes used for hypothesis testing [15]. For the fingerprinting protocol, the case of unequal inputs would hold the laxer promise of the visibility $\mathcal{V}_{2} \leq 0.56$. The shadowed gray region in Fig. 4(c) indicates the range of error values obtained from Monte Carlo simulated photon count statistics with $\mathcal{V}_{1}=$ 0.98 and $0 \leq \mathcal{V}_{2} \leq 0.56$, and processed using the NeymanPearson test designed for $\mathcal{V}_{2}=0.56$. It is seen that the decision rule works also in this more general scenario.

Let us close by discussing the parameter regime required to demonstrate quantum advantage for the fingerprinting protocol based on the primitive presented here. For input data sets $n$ bits long, in the classical scenario it is necessary to reveal at least $O(\sqrt{n})$ bits of information [17]. As shown in the Supplemental Material [16], in the absence of an external phase reference the strategy presented here makes it possible to maintain the exponential enhancement in the number of revealed bits scaling as $O\left(\log _{2} n\right)$, analogously to the coherent protocol [5]. For the error probability $\varepsilon=$ $10^{-4}$ our protocol beats the best currently known classical protocol [18] for $n \geq 2.3 \times 10^{5}$ and the ultimate classical limit [10] for $n \geq 6.3 \times 10^{8}$ bits. It is assumed here that for identical inputs the deviation of the visibility $\mathcal{V}_{1}=0.98$ from 1 stems from experimental imperfections, while unequal inputs are guaranteed to produce maximum 
visibility $\mathcal{V}_{1}=0.56$ with the same contribution from imperfections. In this scenario the attainable code rate for mapping input data sets onto binary phase patterns is $R=0.12$, which implies that the quantum advantage can be observed for pattern lengths exceeding $1.9 \times 10^{6}$ and $5.2 \times 10^{9}$ to beat the best known classical protocol and the classical limit, respectively. If the optical signals are modulated with the $100 \mathrm{GHz}$ bandwidth achievable with standard $\mathrm{LiNbO}_{3}$ electro-optic modulator technology [19], one would require laser sources correspondingly with a kilohertz or a few-hertz linewidth to ensure phase stability over the signal duration. While the former requirement can be met by commercial single-frequency lasers, in the latter case more sophisticated, yet available, laser systems would be needed [20,21].

In conclusion, we described and verified experimentally a strategy to identify the modal overlap between two optical signals with a random relative phase using higher-order interference. It can be viewed as an implementation primitive for a number of quantum-enhanced protocols, when a shared phase reference is not available. As illustrated by the quantum fingerprinting example, this approach offers an analogous scaling advantage compared to classical protocols as schemes utilizing first-order coherence. The experimental demonstration of the quantum advantage should be within the reach of current technology.

We thank E. Kashefi, N. L. Lütkenhaus, F. Xu, and Q. Zhang for insightful discussions. This work was supported by the Foundation for Polish Science under the TEAM project "Quantum Optical Communication Systems" cofinanced by the European Union under the European Regional Development Fund. M. J. was supported by the Foundation for Polish Science.

*michal.jachura@ fuw.edu.pl

[1] G. Brassard, Found. Phys. 33, 1593 (2003).

[2] H. Buhrman, R. Cleve, S. Massar, and R. de Wolf, Rev. Mod. Phys. 82, 665 (2010).

[3] D. Gottesman and I. Chuang, arXiv:quant-ph/0105032.
[4] H. Buhrman, R. Cleve, J. Watrous, and R. de Wolf, Phys. Rev. Lett. 87, 167902 (2001).

[5] J. M. Arrazola and N. Lütkenhaus, Phys. Rev. A 89, 062305 (2014).

[6] J. M. Arrazola and N. Lütkenhaus, Phys. Rev. A 90, 042335 (2014).

[7] P. L. Clarke, R. Collins, V. Dunjko, E. Andersson, J. Jeffers, and G.S. Buller, Nat. Commun. 3, 1174 (2012).

[8] R. J. Collins, R. J. Donaldson, V. Dunjko, P. Wallden, P. J. Clarke, E. Andersson, J. Jeffers, and G. S. Buller, Phys. Rev. Lett. 113, 040502 (2014).

[9] F. Xu, J. M. Arrazola, K. Wei, W. Wang, P. Palacios-Avila, C. Feng, S. Sajeed, N. Lütkenhaus, and H. K. Lo, Nat. Commun. 6, 8735 (2015).

[10] J.-Y. Guan, F. Xu, H.-L. Yin, Y. Li, W.-J. Zhang, S.-J. Chen, X.-Y. Yang, L. Li, L.-X. You, T.-Y. Chen, Z. Wang, Q. Zhang, and J.-W. Pan, Phys. Rev. Lett. 116, 240502 (2016).

[11] R. T. Horn, S. A. Babichev, K.-P. Marzlin, A. I. Lvovsky, and B. C. Sanders, Phys. Rev. Lett. 95, 150502 (2005).

[12] J. Du, P. Zou, X. Peng, D. K. L. Oi, L. C. Kwek, C. H. Oh, and A. Ekert, Phys. Rev. A 74, 042319 (2006).

[13] M. Jachura, M. Lipka, M. Jarzyna, and K. Banaszek, Opt. Express 25, 27475 (2017).

[14] T. M. Cover and J. A. Thomas, Elements of Information Theory (Wiley, New York, 1991).

[15] R. R. Bahadur and R. R. Rao, Ann. Math. Stat. 31, 1015 (1960).

[16] See Supplemental Material at http://link.aps.org/ supplemental/10.1103/PhysRevLett.120.110502 for details.

[17] A. Ambianis, Algorithmica 16, 298 (1996).

[18] L. Babai and P. G. Kimmel, Randomized simultaneous messages: solution of a problem of Yao in communication complexity, in Proceedings of Computational Complexity. Twelve Annual IEEE Conference (IEEE, New York, 1997), pp. 239-246.

[19] K. Noguchi, O. Mitomi, and H. Miyazawa, J. Lightwave Technol. 16, 615 (1998).

[20] M. Notcutt, L.-S. Ma, J. Ye, and J. L. Hall, Opt. Lett. 30, 1815 (2005).

[21] H. Stoehr, F. Mensing, J. Helmcke, and U. Sterr, Opt. Lett. 31, 736 (2006). 